\section{An alien in the gallbladder. A rare case of biliary ascariasis in an Italian emergency department}

\section{Enrico Ferri, Angelo lanni, Laura Magrini, Salvatore Di Somma}

Emergency Department, S. Andrea

Hospital, Rome, Italy

\section{Abstract}

Ascariasis is a common infection in many developing countries. The prevalence of ascariasis is related to poverty, poor hygienic and sanitary conditions. The adult form of Ascaris lumbricoides usually resides in the human intestinal lumen (more frequently in the jejunum and middle ileum) and does not cause symptoms. However, it can occasionally cause severe complications such as intestinal obstruction or perforation peritonitis. Its migration into the biliary tract is not uncommon but gallbladder involvement is very rare. Abdominal ultrasonography is essential to detect the presence of this parasite. In this article, we describe the radiologic findings, clinical manifestations and successful medical treatment of a patient with gallbladder ascariasis diagnosed in an emergency setting.

\section{Introduction}

Ascaris lumbricoides (AL) is one of the most common parasitic infections of human gastrointestinal tract. ${ }^{1}$ Humans are permanent hosts and they are often asymptomatics. Ascaris lumbricoides, which usually resides in the small bowel, may migrate into aberrant sites, overall into the bile duct, causing biliary colic and obstructive jaundice. ${ }^{2}$ Ascaris lumbricoides adult worm, typically $15-30 \mathrm{~cm}$ long and 3-6 mm thick, ${ }^{2}$ rarely settles in the gallbladder ${ }^{3-}$ ${ }^{5}$ due to the anatomical characteristics of the cystic duct which is narrow and tortuous. Anomalous origin of the cystic duct directly from the papilla of Vater ${ }^{6}$ and previous sphinterotomy ${ }^{7}$ facilitate worm invasion into the gallbladder. Pregnant women may be more susceptible due to relaxant effect of hormones on the smooth muscle of the bile ducts. ${ }^{8}$

Ultrasonography (US) is an important noninvasive diagnostic procedure in the work-up of these patients, ${ }^{4,9,10}$ which usually describe a clinical picture suggesting a gallstone disease. It is one of the most used diagnostic tools in the emergency department (ED) because it is safe, not invasive, rapid to perform and, consequently avoids wasting of time for the diagno- sis and the treatment of several pathological conditions in the ED. Conservative treatment with anthelminthic agents is the first line therapy; no response to medical therapy usually indicates the need for endoscopic or surgical interventions. ${ }^{7,10-14}$

\section{Case Report}

An 88-year-old Italian male was admitted to our ED with a ten-day history of recurrent abdominal pain in the right upper abdomen. He had history of hypertensive disease, benign prostatic hypertrophy, and he had an implanted pacemaker device. He denied previous episodes of gallstone disease, abdominal trauma or previous surgery. He was a farmer living in the rural zone of the suburbs of Rome.

Physical examination revealed tenderness in right hypochondrium. Laboratory tests performed at admission in ED showed: aspartate transaminase $14 \mathrm{U} / \mathrm{L}$, alanine transaminase 11 U/L, alkaline phosphatase $52 \mathrm{U} / \mathrm{L}$, gamma glutamyl transpeptidase $12 \mathrm{U} / \mathrm{L}$, bilirubin total/direct $0.48 / 0.11 \mathrm{mg} / \mathrm{dL}$, lactate dehydrogenase $364 \mathrm{U} / \mathrm{L}$, lipase $35 \mathrm{U} / \mathrm{L}$, amylase $40 \mathrm{U} / \mathrm{L}$, hemoglobin $11.4 \mathrm{~g} / \mathrm{dL}$, white blood cells 10,120/L with $5 \%$ eosinophils, platelets $226,000 / \mathrm{L}$ and c reactive protein (CRP) 8.4 $\mathrm{mg} / \mathrm{dL}$.

An abdominal US showed a thickening of the gallbladder's wall with a vermiform nonshadowing image of flukes $(37 \times 23 \mathrm{~mm})$, which showed active motility into its lumen (Figure 1). Electrocardiography and chest X-ray did not showed abnormalities. The proven AL's bowel infection was confirmed by a stool test that revealed the presence of AL's ova. The patient was hospitalized in the ED ward.

To avoid surgical intervention, medical treatment was initially tried as follows: albendazol $400 \mathrm{mg}$ per os, intravenous (i.v.) fluids, pantoprazol $40 \mathrm{mg}$ i.v. and ampicillin/sulbactam $1.5 \mathrm{~g}$ i.v. every $8 \mathrm{~h}$. Analgesic therapy with ketoprophene $160 \mathrm{mg}$ i.v. was also prescribed. Patient got a relief from symptoms after 3 days of conservative treatment and gallbladder US did not show abnormal images anymore.

\section{Discussion}

Ascaris lumbricoides is a common infection in developing countries of world tropical and subtropical regions. ${ }^{1}$ Prevalence of ascariasis is related to poverty, malnutrition, low standard of public health and hygiene. ${ }^{11}$ Globally, 819.0 million people are affected by AL infection; a total 166 countries were classified as potentially endemic, including all countries in Asia, Oceania, Latin America and the
Correspondence: Enrico Ferri, Emergency Department, S. Andrea Hospital, via di Grottarossa 1035, Rome, Italy.

Tel: +39.335.5377453 - Fax: +39.06.33776616.

E-mail: enrico.ferri@ospedalesantandrea.it

Key words: Ascaris lumbricoides; Helminthic infection; Biliary ascariasis; Emergency department.

Contributions: EF wrote the introduction, discussion and conclusions; AI wrote the case report; LM has searched the literature and worked as supervisor; SDS has been involved in supervision.

Conflict of interest: the authors declare no potential conflict of interest.

Received for publication: 8 August 2015 .

Revision received: 10 November 2015.

Accepted for publication: 27 November 2015.

This work is licensed under a Creative Commons Attribution 4.0 License (by-nc 4.0).

(C) Copyright E. Ferri et al., 2016

Licensee PAGEPress, Italy

Emergency Care Journal 2016; 12:5480

doi:10.4081/ecj.2016.5480

Caribbean, North Africa and the Middle East and sub-Saharan Africa. ${ }^{1}$ Frequently, patients with AL infection are asymptomatic. ${ }^{2}$ Gastrointestinal symptoms are typically related to the mechanical effects of high parasite loads.

Ascaris lumbricoides intestinal infection often results in abdominal pain, anorexia, vomiting, diarrhea and impaired absorption. ${ }^{2,4,11,14}$ Sometimes a bolus of worms causes a small-bowel obstruction especially in children where intestinal lumen is small. ${ }^{15}$ Complications including volvulus, gangrene, intestinal perforation massive gastrointestinal bleeding. ${ }^{2} \quad$ Occasionally, worms migrate through the ampulla of Vater into the biliary tree and gallbladder, resulting in bile duct obstruction, obstructive jaundice, single or recurrent cholangitis, colecysthitis, empyema, hepatic abscess and pancreatitis. ${ }^{4,7,14,16,17}$

Ascariasis is considered the second cause of biliary-related symptoms and pancreatitis in endemic regions, ${ }^{2}$ after stone disease. Hepatobiliary ascariasis is more common now than in the past, maybe because of the more frequent use of ultrasonography and endoscopic diagnostic procedures in the clinical practice., ${ }^{5,9,11}$

Sonographic findings included a non-shadowing, long, echogenic structure in the form of a coil, an echogenic strip with central anechoic tube, an echogenic structure extending across the gallbladder giving it a septate appearance, and characteristic erratic, non-directional, zigzag movements of these echogenic struc- 


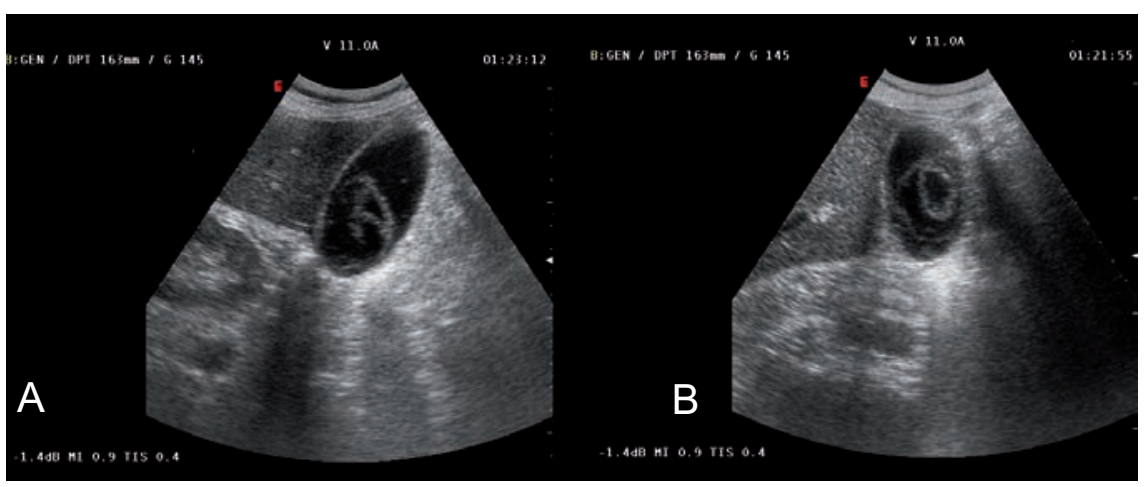

Figure 1. Ultrasonography of the abdomen showing echogenic, tubular, coiling lines inside the lumen of the gallbladder (A) with characteristic erratic, non-directional, zigzag movements (B).

tures in the gallbladder lumen., ${ }^{3,4,9,10,18}$ Computed tomographic scanning ${ }^{10,12}$ and magnetic resonance imaging (MRI) ${ }^{19}$ may also be used to identify worm(s) in the liver or bile ducts but this is not usually necessary. Ultrasonography promptly shows worm's movement in the biliary tree and this is an important advantage of US over CT and MRI., ${ }^{42}$

Endoscopic retrograde cholangiograms may be used for the diagnosis of hepatobiliary ascariasis showing a long tubular filling defect in the bile duct or gallbladder and it is useful for therapeutic purposes allowing the direct removal of the worm. ${ }^{7}$ Therefore, the endoscopic procedure should be restricted to those cases in which US is technically inadequate or when it is impossible to obtain a diagnosis. ${ }^{14}$ Moreover, it is not used in emergency settings. Several reports exist about the successful medical treatment of gallbladder ascariasis., ${ }^{5,810,12-}$ $14,18,19$ Sometimes surgery ${ }^{4,6,8,10,16}$ or the endoscopic procedures ${ }^{7,8,10}$ such as endoscopic retrograde cholangiopancreatographic examination are required. Albendazole and mebendazole are first-line agents for the treatment of ascariasis. ${ }^{20}$

\section{Conclusions}

Although gallbladder ascariasis is more commonly detected in developing tropical and subtropical countries, clinicians need to be aware about this disease because of its possible spreading due to world travel, population migration and climate changing. Ultrasono- graphy is an important, simple, cheap, safe and reliable and non-invasive diagnostic procedure in the evaluation of the patient with gallbladder ascariasis who usually arrives to ED with a clinical picture suggestive of gallstone disease. Other imaging examinations are rarely needed. Gallbladder ascariasis can be treated medically. Unless an associated disease is present, symptoms persist or a progressive complication arises, the medical treatment should be the first choice before endoscopy or surgery.

\section{References}

1. Pullan RL, Smith JL, Jasrasaria R, Brooker SJ. Global numbers of infection and disease burden of soil transmitted helminth infections in 2010. Parasite Vector 2014;7:37.

2. Khuroo MS. Ascariasis. Gastroenterol Clin N 1996;25:553-77.

3. Khuroo MS, Zargar SA, Yattoo GN. Sonographic findings in gallbladder ascariasis. J Clin Ultrasound 1992;20:58791.

4. Javid G, Wani N, Gulzar GM, et al. Gallbladder ascariasis: presentation and management. Brit J Surg 1999;86:1526-7.

5. Gönen KA, Mete R. A rare case of ascariasis in the gallbladder, choledochus and pancreatic duct. Turk J Gastroenterol 2010;21:454-7.

6. Majid Z, Masood I, Pirzada MT. Ascaris lumbricoides and its invasion of the accessory cystic duct: an unusual presentation. Trop Doct 2015;45:129-31.

7. Sandouk F, Haffar S, Zada MM, et al. Pancreatic-biliary ascariasis: experience of 300 cases. Am J Gastroenterol 1997; 92:2264-7.

8. Shah OJ, Robanni I, Khan F, et al. Management of biliary ascariasis in pregnancy. World J Surg 2005;29:1294-8.

9. Filice C, Marchi L, Meloni C, et al. Ultrasound in the diagnosis of gallbladder ascariasis. Abdom Imaging 1995;20:320-2.

10. Mani S, Marchant H, Sachdev R, et al. Sonographic evaluation of biliary ascariasis. Australas Radiol 1997;41:204-6.

11. Das AK. Hepatic and biliary ascariasis. J Glob Infect Dis 2014;6:65-72.

12. Cha DY, Song IK, Choi HW, et al. Successful elimination of Ascaris lumbricoides from the gallbladder by conservative medical therapy. J Gastroenterol 2002;37:758-60.

13. Misra MK, Singh S, Bhagat TS. Successful elimination of Ascaris lumbricoides from the gallbladder by conservative medical therapy. Indian J Surg 2013;75(Suppl.1): 379-81.

14. González AH, Regalado VC, Van den Ende J. Non-invasive management of Ascaris lumbricoides biliary tact migration: a prospective study in 69 patients from Ecuador. Trop Med Int Health 2001;6:14650.

15. Wani I, Rather M, Naikoo G, et al. Intestinal ascariasis in children. World J Surg 2010;34:963-8.

16. Elaldi N, Turan M, Arslan M, et al. An unusual cause of cholecystitis: a worm in the bag. Emerg Med J 2003;20:489-90.

17. Lee TH, Park SH, Lee CK, et al. Ascaris lumbricoides-induced acute pancreatitis. Gastrointest Endosc 2012;75:192-3.

18. Khanduri S, Parashari UC, Agrawal D, Bhadury S. Ascariasis of gallbladder: a rare case report and a review of the literature. Trop Doct 2014;44:50-2.

19. Mittal G, Gupta R, Mittal P. Magnetic resonance demonstration of gall bladder ascariasis. Indian J Gastroenterol 2012;31: 42.

20. Keiser J, Utzinger J. Efficacy of current drugs against soil-transmitted helminth infections: systematic review and metaanalysis. J Am Med Assoc 2008;299:193748 . 